\title{
New problems of optimal path coordination for multi-vehicle systems
}

\author{
J. Borges de Sousa, J. Estrela da Silva and F. Lobo Pereira
}

\begin{abstract}
New problems of optimal path coordination for multiple vehicles are introduced, formulated and solved in the framework of dynamic optimization. The novelty of these problems arises in several ways. The cost function and the dynamics include non-trivial dependencies, modeled through existential quantification over groups of vehicles - this leads to non-Lipschitz behavior and to non-standard optimal control problems. There are consumable resources, modeled with the help of integral constraints - the structure of the constraints suggested new strategies for optimal cooperation which outperform the results obtained with standard formulations with state-constraints. Our formulation uses the structure of the problem to decouple the overall optimization into simpler coupled problems in lower-dimensional spaces. This is expressed in the form of the solution, which is encoded as the composition of value functions in lower-dimensional spaces.
\end{abstract}

\section{INTRODUCTION}

Problems of optimal path coordination for multiple vehicles are posing new challenges to control. Consider, as an example, the problem of controlling operations of unmanned air vehicles $v_{1}$ (UAV) in hostile air spaces. The probability of survival of an UAV is directly proportional to the value of the path integral taken with respect to some risk function [1]; the level of risk is significantly reduced when the UAV flies under the protection of an UAV $v_{2}$ carrying a jamming device. This is an example of a collaborative control problem where vehicles coordinate their paths to improve individual or group performance. Other examples include the use of bulldozers (or icebreakers) to open routes for other cars (or ships) and air refueling missions. The bulldozer modifies or "morphs" the terrain (the cost function shared by other vehicles using the road). The questions are: What is the value of cooperation? Is cooperation useful at all? If so, in what extent? How should the vehicles cooperate in an optimal fashion under budget (fuel) constraints?

We discuss these questions with the help of a simple twovehicle optimal path coordination control problem. This is a non-standard optimal control problem, in the sense that the the actions of one dynamic system (bulldozer) change the cost function for the others (cars) in a non-Lipschitz way. A basic form of this problem was studied in [2]. The problem is revisited here and new features are incorporated: physical obstacles and the possibility of choosing the optimal

This work partially funded by Fundação da Ciência e Tecnologia and by the EU under the project Control for Coordination.

J. Borges de Sousa and F. Lobo Pereira are with the Electrical and Computer Engineering Department, Faculty of Engineering, Porto University, R. Dr. Roberto Frias, s/n 4200-465 Porto, Portugal jtasso, flpefe.up.pt

J. Estrela da Silva is with the Electrical Engineering Department, Porto Polytechnic Institute, Rua Dr. António Bernardino de Almeida 431,4200072 Porto, Portugal jes@isep.ipp.pt departure point of $v_{1}$ among several candidate regions. We also relax some of the assumptions in order to make the formulation applicable to a broader class of systems. Like in [2] we formulate the collaborative control problem for the two vehicles $v_{1}$ and $v_{2}$ as an optimal control problem for a hybrid automaton with three discrete states (the hybrid automaton models the combinatorial aspects of the problem). We follow the hybrid systems model from [3].

We tackle the problem in the framework of dynamic programming (DP) [4]. DP approaches the problem of optimizing the behavior of a dynamic system with respect to some cost function by introducing a value function which gives, at each point of the state space, the optimal cost to go for the system. This has the advantage of providing a global perspective on the optimal behavior of the system, as also of facilitating the development of optimal feedback control laws. For general nonlinear problems, the value function is obtained from the solution of a Hamilton-Jacobi-Bellman (HJB) partial differential equation (PDE). In this work we pursue that path and we employ a numerical procedure for the solution of the HJB PDE.

The problem described here combines the following aspects: a) both controlled (discrete input) and autonomous (state dependent) transitions between discrete states are allowed; b) controlled transition may be triggered only at certain points of the continuous state space; c) running cost depends on the discrete state; d) nonlinear continuous dynamics are assumed. We are not aware of any published work on DP for hybrid systems combining all of the above mentioned aspects and offering a general efficient computation method. In [3], where the author presented a taxonomy for hybrid systems which subsumed previous models, optimal control of hybrid systems in the setting of DP is discussed. However, no general efficient computation method is devised there. That line of development is still an open problem today. Most of the results toward efficient methods are obtained by restricting their range of applicability just to certain classes of problems. In [5] the authors develop a Hybrid Bellman Equation for systems with regional dynamics (i.e., only autonomous transitions are considered). The results are demonstrated only for problems featuring two dimensional continuous dynamics, with quadratic cost. Practical applicability of the method to more complex systems is not discussed. A similar problem for piecewise affine systems is presented in [6], also using ideas from DP. In [7] the authors use stochastic DP for a related collaborative control problem. There is a vast body of work on dynamic optimization of switched systems and multi-model systems (switched systems with state jumps) but none seems to meet all the 
above mentioned aspects (see [8], [9], [10] and references therein). In [11], the authors describe a single pass numerical method for the solution of the HJB PDE in hybrid optimal control problems. We use some insights of that work in our numerical example.

The paper is organized as follows. In section II we state and formulate a path coordination problem in the framework of hybrid systems. In section III we use DP techniques to characterize the solution to the problem. In section IV we discuss optimal strategies in the framework of DP and in section $\mathrm{V}$ we illustrate practical application of the approach using numerical examples. In section VI we draw the conclusions.

\section{PROBLEM FORMULATION}

\section{A. The system}

We need some definitions. $U A V$ is a generic term for the entities composing our force. There is a finite set of types, called UAVTypes $=\{$ simple, jammer $\}$. A UAV is characterized by its type and its (two-dimensional) location $(x)$. So, our force with $N$ UAVs is thus described by a set of the form

$$
U A V s=\left\{v_{1}=\left(\text { type }_{1},\left(x_{1}\right)\right), \cdots, v_{N}=\left(\text { type }_{N},\left(x_{N}\right)\right)\right\} .
$$

Consider the simplest problem setting with $N=2$, type $_{1}=$ simple and type $_{2}=$ jammer. Vehicle $v_{1}$ has to find the optimal trajectory from $\alpha$ to $\gamma$. The instantaneous path cost for $v_{1}$ is reduced by a fixed amount $l$ when the position of this vehicle "coincides" with the position of another vehicle, $v_{2}$; this means that the path cost for $v_{1}$ is a discontinuous function of the relative positions of the two vehicles. $v_{2}$ has a limited amount of fuel; it departs from $\beta \neq \alpha$ and is required to return to $\beta$ before it runs out of fuel. The corresponding motion models are

$$
\begin{aligned}
\dot{x}_{i}(t) & =f_{i}\left(x_{i}, u_{i}\right), x_{i} \in \mathbb{R}^{n}, u_{i} \in U_{i}, t \geq 0 \\
x_{1}(0) & =\alpha, x_{2}(0)=\beta
\end{aligned}
$$

where $u_{i}$ are the controls and $U_{i}$ are closed sets. The fuel consumption of $v_{2}$ is modeled by the state variable $c_{2} \in \mathbb{R}$

$$
\begin{aligned}
\dot{c_{2}}(t)=g_{2}\left(x_{2}, u_{2}\right) & = \begin{cases}w_{2}\left(x_{2}, u_{2}\right) & \text { if } c_{2}>0 \\
0 & \text { otherwise }\end{cases} \\
c_{2}(0) & =\theta
\end{aligned}
$$

where $w_{2}(.,) \leq$.0 .

Consider $v_{1}$. The cost of a path joining $\alpha$ and $\gamma$ is

$$
J_{1}\left(u_{1}(.), \ldots, u_{N}(.), \gamma\right)=\int_{0}^{t_{f}} l\left(x_{1}\right) \cdot k_{1}\left(x_{1}, u_{1}\right) d s
$$

where $k_{1}(.,)>0,. t_{f}$ is the first time when $x_{1}\left(t_{f}\right)=\gamma$ under the control functions $u_{1}(),. \ldots u_{N}($.$) and l: \mathbb{R}^{n} \rightarrow[0,1]$ is

$$
l\left(x_{1}\right)= \begin{cases}\xi & \text { if } \exists v_{i} \in U A V s: x\left(v_{i}\right)=x_{1} \wedge \text { type }\left(v_{i}\right)=\text { jammer } \\ 1 & \text { otherwise }\end{cases}
$$

The function $l$ models the fact that the path cost for $v_{1}$ is reduced $(\xi \in(0,1))$ when the position of $v_{1}$ coincides with the position of another $U A V$.
The standing assumptions are:

A1) $f_{i}: \mathbb{R}^{n} \times U_{i} \rightarrow \mathbb{R}^{n}$ and $w_{2}: \mathbb{R}^{n} \times U_{i} \rightarrow$ $\mathbb{R}$ are uniformly Lipschitz in $x$ and uniformly continuous in the control variable. This condition ensures existence and uniqueness of solutions for the differential equations.

A2) There exist $K_{1}<\infty$ and $1 \leq \varsigma_{1}<\infty$ such that $\left\|l\left(x_{1}, x_{2}\right) \cdot k_{1}\left(x_{1}, u_{1}\right)\right\| \leq K_{1}\left(1+\left\|\left(x_{1}, x_{2}\right)\right\|\right)^{\varsigma_{1}}$ for $\left(x_{1}, x_{2}\right) \in \mathbb{R}^{n} \times \mathbb{R}^{n}, u_{1} \in U_{1}$.

A3) There exist $K_{2}<\infty$ and $1 \leq \varsigma_{2}<\infty$ such that $\left\|g_{2}\left(x_{2}, u_{2}\right)\right\| \leq K_{2}(1+\|x\|)^{\varsigma_{2}}$ for $x \in \mathbb{R}^{n}, u_{2} \in$ $U_{2}$. This assumption and the previous are related to the existence of solution to the problem.

A4) $0 \in$ int $f_{i}\left(x_{i}, U_{i}\right)$ (locally controllable).

A5) $f_{1}\left(x, U_{1}\right) \subseteq f_{2}\left(x, U_{2}\right)$. This means that $v_{2}$ is capable of replicating the motions of $v_{1}$. If necessary, this constraint can be enforced by considering a new set of controls for $v_{1}, U_{1}^{c} \subset U_{1}$, such that $f_{1}\left(x, U_{1}^{c}\right) \subseteq f_{2}\left(x, U_{2}\right)$.

A6) The vehicles are allowed to meet only once and then move together up to the point where $v_{2}$ returns to $\beta$ (this precludes behaviors where the vehicles move together and separate repeatedly).

A7) $k_{1}\left(x_{1}, u_{1}\right)$ may take an arbitrary high value (finite, so that A2 is fulfilled), in the line of the exact penalization method (see [12]). This is done to define forbidden regions, which may be used to represent, for instance, physical obstacles.

\section{B. The case for coordination}

Consider that $v_{1}$ is operating in isolation $(l=1)$

Problem 1: [Uncoordinated] Find

$$
\inf _{u_{1}(.)} J_{1}\left(u_{1}(.), \gamma\right)
$$

The path planning problem becomes more interesting when the two vehicles are allowed to coordinate their motions. It may be worthwhile for $v_{1}$ to deviate from the optimal path for Problem 1 to join $v_{2}$ before reaching $\gamma$. The following example illustrates this point.

Example 2.1: Consider Figure 1. Let: $x_{i} \in \mathbb{R}^{2}, \dot{x}_{i}(t) \in B_{0}, i=1,2$ ( $B_{0}$ is the closed unit ball). $\alpha=(0,0), \beta=(50,40), \gamma=(100,0)$. $\eta=(39.2000,24.1254), \mu=(60.7999,24.1254)$. $c_{2}(0)=\theta=12$. $k_{1}\left(x_{1}, u_{1}\right)=1,-w_{2}\left(x_{2}, u_{2}\right)=0.2, l(x, x)=0.1$.

The circle of radius 30 centered at $\beta$ encloses the set of points reachable by $v_{2}$ within fuel constraints. This will be discussed in more detail later. In this example, the fuel optimal paths for $v_{2}$ are straight lines. The same happens with the optimal paths for $v_{1}$ (for fixed values of $l$ ). This is because we have simple dynamics and piecewise constant cost functions. The straight line joining $\alpha$ and $\gamma$ is the optimal path for Problem 1; the optimal cost is 100. The cost of the path $(\alpha, \eta, \mu, \gamma)$, where $v_{1}$ deviates from the original optimal path to benefit from a cost reduction in the segment $(\eta, \mu)$, is $94.2182 . v_{2}$ complies with the constraints by taking 


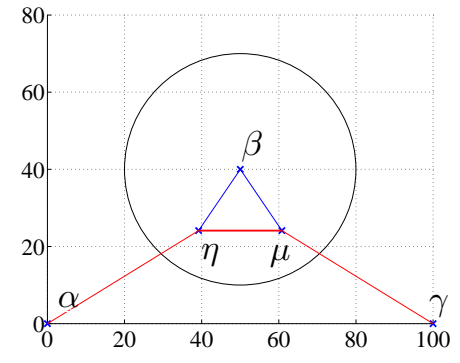

Fig. 1. Example of coordinated paths.

a loop (triangle) from $\beta$, with fuel cost 12.0000 (within the fuel budget).

The optimal coordinated path planning problem for $v_{1}$ is Problem 2: Find

$$
\inf _{u_{1}(.), u_{2}(.)} J_{1}\left(u_{1}(.), u_{2}(.), \gamma\right)
$$

$\inf _{u_{1}(.), u_{2}} J_{1}\left(u_{1}(),. u_{2}(),. \gamma\right) \in \mathbb{R}$ under the stated assumptions

Lemma 2.1: Let $R$ denote the set of points reachable by $v_{2}$ for a round trip from $\beta$ under fuel budget $\theta$. Joint operation may only happen on $R$.

A characterization of $R$ is in order. For that purpose, we introduce the following value functions: $V_{2 b}(x)$, a map of $x$ to the minimum amount of fuel required to reach $x$ after departing from $\beta$; and $V_{2 c}(x)$, a map of $x$ to the minimum amount of fuel required to reach $\beta$ after departing from $x$. These functions are not necessarily the same. For instance, if the vehicle has to face directional winds, the expended fuel will be different depending on the direction of the trip. Details on dynamic optimization techniques for reachability analysis can be found in see [13].

On example 2.1, the fuel consumption of $v_{2}$ depends only on the trip duration (as given by $w_{2}$ ); $v_{2}$ will adopt its maximum velocity (unit velocity) in order to minimize the trip duration, which is given by $\frac{\|x-\beta\|_{2}}{1}$. Due to the simple dynamics of $v_{2}$ it is trivial to see that $V_{2 b}(x)=V_{2 c}(x)=$ $0.2\|x-\beta\|_{2}$. Therefore, $R$ is the circle of radius 30 with center $\beta$.

Remark 1: Notice that was $v_{2}$ faster than $v_{1}$ it could use that velocity to reach farther points without breaking A5.

\section{The structure of the optimal solution}

The existential quantifier in the cost function for $v_{1}$ (see (2)) means that it depends on endogenous and exogenous variables. There is a continuous dependence on the endogenous variables (the position and controls of $v_{1}$ ) and a discontinuous dependence on the exogenous variables the position of other vehicles ( $v_{2}$ in our problem). The discontinuity on the position of $v_{2}$ means that the cost function does not provide information on what $v_{2}$ should do when the two vehicles are not moving together. In our approach, that behavior is implicitly imposed by $V_{2 b}(x)$ and $V_{2 c}(x)$, as explained below. In fact this discontinuity introduces a combinatorial aspect to the problem (for the case of more vehicles). The discontinuous behavior can be described by enumerating all possible interactions. For the two vehicle problem this could be modeled with two discrete states. However, the requirement that the vehicles can only meet once demands an additional state to memorize the fact that a meeting took place.

Assuming that the optimal solution implies coordinated operation, $v_{2}$ has to travel a round trip from $\beta$ to meet with $v_{1}$ at some some point in $R$. The round trip is composed of three path segments: $\left(\beta, x_{b}\right),\left(x_{b}, x_{c}\right),\left(x_{c}, \beta\right) .\left(x_{b}, x_{c}\right)$ is the path traveled together by $v_{1}$ and $v_{2} ; c_{b}, c_{c}$ and $c_{f}$ are the levels of fuel available for $v_{2}$ at respectively $x_{b}, x_{c}$ and $\beta$. We observe that the points in the path segment $\left(x_{b}, x_{c}\right)$ are in $R$.

If the optimal path for $v_{1}$ in Problem 2 goes through $R$, then both vehicles go through discrete states $(a, b, c)$ as follows. $v_{1}$ starts at state $a$ to follow the optimal path connecting $\alpha$ and $x_{b} \in R$; in state $b$, it is accompanied by $v_{2}$ in the optimal path connecting $x_{b}$ to $x_{c}$; and in state $c$ it follows the optimal path connecting $x_{c}$ to $\gamma$.

When the two vehicles meet at point $x_{b}$, the path optimization for both vehicles is no longer decoupled. Moreover, the model of fuel consumption for $v_{2}$ adds an integral constraint to the problem. From the perspective of $v_{1}$, all that really matters in what concerns $v_{2}$ is: 1) the point where the meeting takes place; and 2) the amount of the fuel remaining in the fuel tank of $v_{2}$.

In the discrete states $a$ and $c, v_{2}$ follows fuel optimal paths connecting respectively $\beta$ to $x_{b}$ and $x_{c}$ to $\beta$. In order to maximize the coverage of $v_{1}$ by $v_{2}$ we will have $c_{b}=V_{2 b}\left(x_{b}\right)$, since there is no point in $v_{2}$ spending more fuel than needed to reach $x_{b}$.

In what concerns $c_{c}$, we can conclude the following:

Proposition 2.1: Let $p$ be an optimal path for Problem 2 with a segment $\left(x_{b}, x_{c}\right)$ in $R$. Let $V_{2 b c}\left(x, x_{0}\right)$ be the fuel required for $v_{2}$ to follow $v_{1}$ in its optimal path from $x_{0}$ to $x$. Assume that $v_{2}$ is fuel constrained, i.e., that $v_{2}$ has not enough fuel to cover the entire path $p$ Then $c_{c} \geq V_{2 c}\left(x_{c}\right)$, i.e., for certain system dynamics, $v_{2}$ may have to return to $\beta$ with nonzero fuel slack. If $V_{2 b}(x), V_{2 c}(x)$ and $V_{2 b c}\left(x, x_{0}\right)$ are continuous then $c_{c}=V_{2 c}\left(x_{c}\right)$ (zero fuel slack).

We do not present the proof here due to space limitations.

Remark 2: Notice that, in all of the above, we do not imply anything about the way $x_{b}$ is chosen. In what concerns $x_{c}$ we can infer the following corollary: $c_{2}(t) \geq V_{2 c}\left(x_{1}(t)\right) \wedge$ $c_{2}\left(t^{+}\right)<V_{2 c}\left(x_{1}\left(t^{+}\right)\right) \Rightarrow x_{c}=x_{1}(t)$.

\section{Hybrid model}

The formulation of the coordinated optimal path planning problem for vehicle $v_{1}$ requires the consideration of a state variable that keeps track of what each vehicle does. We do this with a 3-state hybrid automaton. The hybrid state space is $S=\bigcup_{v \in\{a, b, c\}}\left(S_{v} \times v\right) . v_{1}$ evolves in $S_{a}=\mathbb{R}^{n}$ after departing from $\alpha$. The positions of the two vehicles coincide in the discrete state $b$. We need an additional variable to keep track of the fuel consumption for $v_{2}$; this is why $S_{b}=$ 
$\mathbb{R}^{n} \times \mathbb{R}_{0}^{+} \cdot v_{1}$ moves in $S_{c}=\mathbb{R}^{n}$ after taking the transition from discrete state $b$ to discrete state $c$ (after leaving $v_{2}$ ).

There is a controlled vector field $f_{v}$ associated to each discrete state, where $f_{a}=f_{c}=f_{1}$ and $f_{b}=\left\{f_{1}, g_{2}\right\}$. The control constraints are $U_{a}=U_{1}, U_{b}=U_{1} \times U_{2}$ and $U_{c}=U_{1}$. In the terminology of [3], associated to each discrete state $v$ there are autonomous jump sets $A_{v, v^{\prime}}$, controlled jump sets $C_{v, v^{\prime}}$ and jump destination sets $D_{v, v^{\prime}}$. The trajectory of the system jumps from $S_{v}$ to $S_{v^{\prime}}$ upon hitting the autonomous jump set $A_{v, v^{\prime}}$; it may or may not leave $S_{v}$ upon hitting the controlled jump set $C_{v, v^{\prime}}$ and it can leave $S_{v}$ at any point in $C_{v, v^{\prime}}$; the destination of a jump is $D_{v, v^{\prime}}$.

In what follows, $x^{i}$ represents the $\mathrm{i}$-th component of $x$.

The autonomous and controlled jump sets for the system are respectively $A=\bigcup_{v, v^{\prime}} A_{v, v^{\prime}}$ and $C=\bigcup_{v, v^{\prime}} C_{v, v^{\prime}}$. The jump set is $J=A \cup C$. These are given by

$$
\begin{aligned}
& C_{a, b}=R \\
& A_{b, c}=\left\{\left(x^{1}, x^{2}, x^{3}\right): x^{3}=V_{2}\left(x^{1}, x^{2}\right)\right\} \\
& D_{a, b}=\left\{\left(x^{1}, x^{2}, x^{3}\right): x^{3} \geq V_{2}\left(x^{1}, x^{2}\right)\right\} \\
& D_{b, c}=S_{c}
\end{aligned}
$$

The transition maps are

$$
\begin{aligned}
G_{a, b}: C_{a, b} \rightarrow D_{a, b}, G_{a, b}(x) & =\left(x, \theta-V_{2}(x)\right) \\
G_{b, c}: A_{b, c} & \rightarrow D_{b, c}, G_{b, c}(x)=\left(x^{1}, x^{2}\right)
\end{aligned}
$$

The interpretation is as follows. $v_{1}$ starts moving in $S_{a}$; if $x_{1}($.$) enters C_{a, b}$ then it may continue in $S_{a}$, or take a controlled jump to $S_{b}$. In the case of a controlled jump, the transition map $G_{a, b}$ maps the current state of $v_{1}$ to a state extended to include the optimal amount of fuel remaining in $v_{2}$ at the same location after departing from $\beta$ with an initial amount of fuel $\theta$. In $S_{b}$, the positions of the two vehicles coincide; there is an autonomous jump from $S_{b}$ to $S_{c}$ when the trajectory of the system hits $A_{b, c}$. This means that $v_{2}$ had to leave, since there was just enough fuel to go back to $\beta$. The jump relation consists of eliminating the third component of the state. The transition maps imply that $v_{2}$ uses fuel optimal strategies to travel to the meeting point and to reach $\beta$ after leaving $v_{1}$. Figure 2 shows the automaton corresponding to this hybrid system.

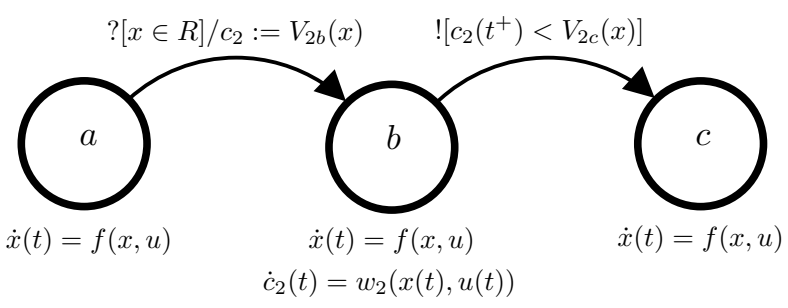

Fig. 2. Hybrid automaton modeling the system. The continuous state space on mode $b$ has one additional dimension to model available fuel in $v_{2}$.

With the aid of the hybrid system formulation, we define $T$ as the set of points reachable by $v_{2}$ in $S_{b}$ under the fuel constraint $\theta$ for a round-trip from $\beta . T$ is the set of all $\left(x^{1}, x^{2}, x^{3}\right) \in S_{b}$ such that the first two components $\left(x^{1}, x^{2}\right)$ are in $R$ and the last component $\left(x^{3}\right)$ satisfies the fuel constraint:

$$
\begin{aligned}
& T=\left\{x \in S_{b}:\left(x^{1}, x^{2}\right) \in R \wedge\left(x^{3} \geq V_{2}\left(x^{1}, x^{2}\right)\right) \wedge\right. \\
& \left.\quad\left(\left(\theta-V_{2}\left(x^{1}, x^{2}\right)\right) \geq x^{3}\right)\right\}
\end{aligned}
$$

Remark 3: $M=\left\{S_{b} \backslash T, b\right\}$ is not reachable in $S$.

\section{DYNAMIC PROGRAMMING}

The precise formulation of the hybrid optimal control problem is presented in [2]. We recover some of the main definitions and results to be used here.

The minimum cost to reach continuous state $x \in \mathbb{R}^{n}$ on discrete state $v \in\{a, b, c\}$, departing from $\alpha$, is defined as $V(x, v)$. In this context, we present a new assumption.

A8) Vehicle $v_{1}$ may choose to leave from any point from a predefined arbitrary set $S_{0} \in \mathbb{R}^{n}$, with initial cost defined by $g(x), x \in S_{0}$.

This means that the boundary condition is given by $\forall x \in$ $S_{0}: V(x, a)=g(x)$. Moreover, $\forall x \in\left(S_{b} \backslash T\right): V(x, b, \sigma)=$ $+\infty$. On example 2.1 we have $S_{0}=\{\alpha\}$ and $V(\alpha, a)=0$.

The following theorems can be proved with the help of the results from [14].

Theorem 1: The value function $V(x, v)$ satisfies the principle of optimality for every $v \in\{a, b, c\}$.

Keep in mind that $v_{1}$ can reach a same position in the three discrete states. The principle of optimality is valid only if the discrete state is also taken in account. For instance, we may have an optimal trajectory from $\alpha$ to $\gamma$, passing through $\eta \in R$ on discrete state $a$, and also an optimal trajectory from $\alpha$ to $\eta$ which is not a subset of the former. That happens because in the later case $\eta$ would be reached on other state than $a$.

Theorem 2: The value function $V(x, v)$ is the viscosity solution of the HJB equation.

$$
\begin{aligned}
V_{t}(x, v)+H\left(x, v, V_{x}\right) & =0,(x, v) \in S \backslash S_{0} \times a \\
V(x, a) & =g(x), x \in S_{0}
\end{aligned}
$$

with

$$
H(x, v, p)=\sup _{u \in U}\left[p(x, v) \cdot f_{v}(x, u)-l(x) \cdot k_{1}(x, u)\right]
$$

\section{OPTIMAL STRATEGIES}

The optimal strategy for $v_{1}$ is derived from the value function $V(x, v)$. This requires some additional computations.

The position of $v_{1}$ is given by the continuous state of the hybrid automaton in the discrete states $a$ and $c$, and by the first two components of the continuous state in the discrete state $b$; the third component, $x^{3}$, is the fuel remaining in $v_{2}$. However, the value function $V$ in $b$ depends not only on the position of $v_{1}\left(x^{1}, x^{2}\right)$, but also on the fuel remaining in $v_{2}$ $\left(x^{3}\right)$. An additional minimization over $x^{3}$ is required. This is done next with the help of a new function, $\tilde{V}: \mathbb{R}^{n} \rightarrow \mathbb{R}$.

$$
\begin{aligned}
\tilde{V}(x, a) & =V(x, a) \\
\tilde{V}(x, b) & =\min _{x^{3} \in\left[V_{2}(x), \theta-V_{2}(x)\right]} V\left(\left(x, x^{3}\right), b\right) \\
\tilde{V}(x, c) & =V(x, c)
\end{aligned}
$$


$\tilde{V}(x, a)$ is also the optimal value function for Problem 1. To find the optimal path cost at $x \in \mathbb{R}^{n}$ we need to drop the dependence of $\tilde{V}$ on the discrete state with another minimization. This is done with the the help of a new function, $\bar{V}(x): \mathbb{R}^{n} \rightarrow \mathbb{R}$.

$$
\bar{V}(x)=\min _{v \in\{a, b, c\}} \tilde{V}(x, v)
$$

The optimal discrete state at the final state of the trajectory $x\left(t_{f}\right)$ is given by

$$
v^{*}=\operatorname{argmin}_{v \in\{a, b, c\}} \tilde{V}\left(x\left(t_{f}\right), v\right)
$$

Observe that $v^{*}$ is not necessarily a singleton. We summarize these observations in the theorem.

Theorem 3: $\bar{V}(\gamma)$ is the optimal value for solving Problem 2. If $v^{*}=a$ then path coordination is not optimal.

The optimal control is given by $u^{*}$ as follows

$$
u^{*}=\operatorname{argmax}_{u \in U}\left[V_{x}(x, v) \cdot f_{v}(x, u)-l(x) \cdot k_{1}(x, u)\right]
$$

\section{NUMERICAL EXAMPLES}

Example 2.1 is an interesting benchmark example because it is relatively simple to validate the associated optimal trajectories geometrically. Even so, the advantages of the approach described in this paper should not be neglected even for an apparently easy problem. This approach allows us to make universal quantification and to answer questions such as "What is the set of destination points for which the optimal trajectory implies vehicle coordination?" (see Theorem 3). A more complete analysis of example 2.1 can be found in [2]. We emphasize that this problem should not be confused with a simple problem of weighted regions (e.g., [15]), since the duration of the coordinated mode depends on the dynamics of $v_{2}$ (namely on its fuel consumption) and its trajectory, not on some predefined boundaries on the continuous statespace.

We start by computing the value function at discrete points of a regular grid $\Omega \subset S$. The value function is computed by numerical methods (described below). Several destination points are then considered. The optimal trajectory to each of those destinations is computed much like as in standard DP problems: by recursive backward in time integration of the system dynamics, with continuous input given by (8). The main differences reside on the need to detect the transitions between discrete states, reverting the state jumps (remember that the computation is performed backward in time) and, of course, selecting the value function accordingly to the discrete state. This procedure is also described below.

For each example we present a figure displaying the level sets of $\bar{V}(x)$, along with the optimal trajectories for arbitrarily selected destination points. The coordinated flight phase is plotted on red (thick). The destinations for which coordinated operation is the optimal choice are filled in gray. For discrete states $a$ and $c$ we use a grid of $400 \times 400$ points. On discrete state $b$ the grid has $400 \times 400 \times 480$ points.

Figure 3 refers to example 2.1. It is possible to see the length of the joint motion path (discrete state $b$ ) varying according to the selected destination point. The computation of $V(x, v)$ took 715 seconds on a computer based on the Intel T7250 processor.

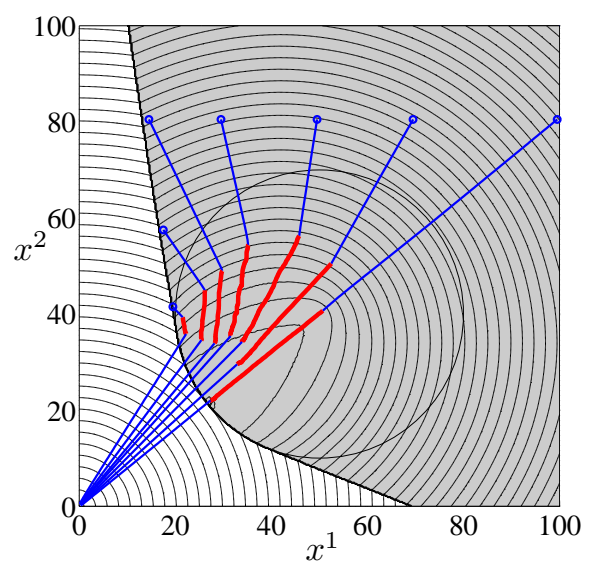

Fig. 3. Level sets of $\bar{V}(x)$ for example 2.1, along with the optimal trajectories for arbitrarily selected destination points. The coordinated flight phase is plotted on red (thick). The circle delimits $R$, the set of points that $v_{2}$ can reach and still return to its initial position. The gray area marks the destinations for which coordinated operation is the optimal choice.

The second example is still based on example 2.1, but with elements that make it harder to compute the optimal trajectories by simple geometrical considerations. First, $v_{1}$ may depart from any point of $S_{0}=\{(0,0)\} \cup\left\{\left(x^{1}, x^{2}\right)\right.$ : $\left.x^{2}=100 \wedge 20 \leq x^{1} \leq 60\right\}$; second, $v_{1}$ is not allowed to reach the rectangular regions $\left\{\left(x^{1}, x^{2}\right):\left(45 \leq x^{1} \leq 55 \wedge\right.\right.$ $\left.\left.0 \leq x^{2} \leq 10\right)\right\},\left\{\left(x^{1}, x^{2}\right):\left(10 \leq x^{1} \leq 38.5 \wedge 85 \leq x^{2} \leq\right.\right.$ $90)\}$ and $\left\{\left(x^{1}, x^{2}\right):\left(42.5 \leq x^{1} \leq 70 \wedge 85 \leq x^{2} \leq 90\right)\right\}$. As illustrated on Figure 4, the computation of the optimal path shows the optimal departing point for $v_{1}$. Also, the implemented numerical algorithm has no problems in dealing with the obstacles. In this case, the computation of $V(x, v)$ took 1115 seconds.

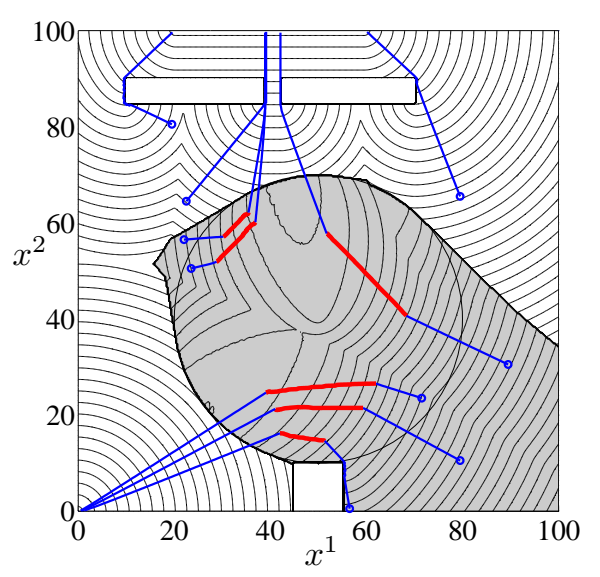

Fig. 4. Level sets of $\bar{V}(x)$ for second example, along with the optimal trajectories for arbitrarily selected destination points. 


\section{A. Algorithms}

The main difficulty in the DP approach for general nonlinear problems is the computation of the solution of the HJB PDE. We perform that task using ideas from [11] and [16]. Those papers describe a class of single-pass numerical algorithm for the static HJB PDE designated as "Ordered Upwind Methods" (OUM). The OUM are inspired by the Dijkstra algorithm, which is characterized by computing the value function in a monotonic fashion, i.e., from the points with lower value to the ones with higher value. This characteristic is important for the efficiency of our approach, namely on handling the controlled transition from $a$ to $b$. However the theoretical analysis of the OUM takes the limiting assumption that $\mathcal{F}(x)=f(x, U)$ is a compact set with the origin in its interior for every $x$ in the continuous state space. Moreover, the performance of the algorithm degrades as $\mathcal{F}(x)$ deviates from an hypersphere centered at the origin. The dynamics considered on the examples presented in this paper fulfill the above mentioned assumption only on states $a$ and $c$. On state $b$, due to the dynamics of the fuel variable, that assumption is not met $(\mathcal{F}(x)$ is a cone with vertex at the origin and axis directed toward decreasing values of $\left.x^{3}\right)$. In order to deal with that case, we made a free adaptation of the ideas of the OUM. The results are consistent with the ones obtained by the "brute force" approach used for example 2.1 in [2]. The current implementation does not follow all hints presented in [16], therefore it might be possible to further improve the computation times mentioned above. As can be seen in the previous subsection, the computation time of the algorithm does not depend solely on the number of grid points. This happens because, at each iteration, the algorithm must manage a front of candidate points and also select the lowest value point from it. The greater complexity of the second example (obstacles, several starting points) leads to a front composed of more points; therefore, the evaluation of the front takes an average time greater than in the first example.

Given $V(x, v)$, the computation of any optimal trajectory takes negligible time. The backward in time integration is performed using the Euler method. The procedure is as follows (remember that $u^{*}(t)$ is given by (8)):

1) Start with $t=0 . x(0)$ is the destination point. Identify the respective optimal discrete state using (7).

2) Check $x(t) \in S_{0}$; if true, stop the procedure.

3) $x(t-\Delta t)=x(t)-\Delta t \cdot f\left(x(t), u^{*}(t)\right)$.

4) If in state $b, c_{2}(t-\Delta t)=c_{2}(t)-\Delta t \cdot w_{2}(x(t))$.

5) $t=t-\Delta t$.

6) If in state $c$, check (7). If the new optimal state is $b$, reset $c_{2}(t)$ to $\operatorname{argmin}_{x^{3}} V\left(\left(x(t), x^{3}\right), b\right)$.

7) If in state $b$, check $\theta-V_{2}(x(t))-c_{2}(t) \leq 0$; if true, switch to state $a$.

8) Go back to step 2

\section{CONCLUSIONS}

We have shown how to use dynamic programming to compute the optimal solution for a class of collaborative control problems. We use the hybrid systems framework to model the problem. This allows a clear description of the logic of the problem and also the consideration of different dimensions for each discrete state, with obvious advantages for computational efficiency. This class of problems features autonomous and controlled transitions between discrete states. We compute a value function for each discrete state. However, it must be remarked that these value functions are coupled, i.e., they may not be computed independently. The solution of the resulting HJB PDE is computed through numerical methods. The global approach allows a systematic qualitative and quantitative determination of whether cooperation is advantageous or not, along with the respective optimal trajectory.

\section{ACKNOWLEDGMENTS}

The authors thank Professor Pravin Varaiya for fruitful discussions and insights.

\section{REFERENCES}

[1] J. B. de Sousa, T. Simsek, and P. Varaiya, "Task planning and execution for uav teams," in Proceedings of the 43rd IEEE Conference on Decision and Control, December 2004.

[2] J. B. de Sousa and J. E. da Silva, "Optimal path coordination problems," in Proceedings of the 47th IEEE Conference on Decision and Control, Cancun, Mexico, December 2008.

[3] M. Branicky, "Studies in hybrid systems: Modeling, analysis and control," Ph.D. dissertation, MIT, 1995.

[4] R. Bellman, Dynamic programming. Princeton University Press, 1957.

[5] A. Schollig, P. Caines, M. Egerstedt, and R. Malhame, "A hybrid bellman equation for systems with regional dynamics," in Proceedings of the 46th IEEE Conference on Decision and Control, Dec. 2007, pp. 3393-3398.

[6] M. Baotic, F. Christophersen, and M. Morari, "Constrained optimal control of hybrid systems with a linear performance index," Automatic Control, IEEE Transactions on, vol. 51, no. 12, pp. 1903-1919, Dec. 2006.

[7] M. Flint, M. Polycarpou, and E. Fernandez-Gaucherand, "Cooperative control for multiple autonomous uavs searching for targets," in Proceedings of the 41st IEEE Conference on Decision and Control. IEEE Control Society, 2002, pp. 2823-28.

[8] H. Axelsson, M. Boccadoro, M. Egerstedt, P. Valigi, and Y. Wardi, "Optimal mode-switching for hybrid systems with varying initial states," Nonlinear Analysis: Hybrid Systems, vol. 2, no. 3, pp. 765 - 772, 2008.

[9] S. Wei, K. Uthaichana, M. Žefran, R. A. DeCarlo, and S. Bengea, "Applications of numerical optimal control to nonlinear hybrid systems," Nonlinear Analysis: Hybrid Systems, vol. 1, no. 2, pp. 264-279, 2007.

[10] C. Seatzu, D. Corona, A. Giua, and A. Bemporad, "Optimal control of continuous-time switched affine systems," IEEE Transactions on Automatic Control, vol. 51, no. 5, pp. 726-741, May 2006.

[11] J. Sethian and A. Vladimirsky, "Ordered upwind methods for hybrid control," in Proceedings of the hybrid systems workshop. SpringerVerlag, 2002, pp. 393-406.

[12] F. H. C. et. al., Nonsmooth Analysis and Control Theory. Springer, 1998.

[13] A. B. Kurzhanskii and P. Varaiya, "Dynamic optimization for reachability problems," Journal of Optimization Theory \& Applications, vol. 108, no. 2, pp. 227-51, 2001.

[14] H. Zhang and M. R. James, "Optimal control of hybrid systems and a systems of quasi-variational inequalities," SIAM Journal of Control and Optimization, vol. 48, no. 2, pp. 722-761, 2006.

[15] J. S. B. Mitchell and C. H. Papadimitriou, "The weighted region problem: finding shortest paths through a weighted planar subdivision," $J$. $A C M$, vol. 38, no. 1, pp. 18-73, 1991.

[16] J. A. Sethian and A. Vladimirsky, "Ordered upwind methods for static hamilton-jacobi equations: Theory and algorithms," SIAM J. Numer. Anal., vol. 41, no. 1, pp. 325-363, 2003. 